\title{
Entanglement creation in a quantum-dot-nanocavity system by Fourier-synthesized acoustic pulses
}

\author{
Ralf Blattmann, ${ }^{1,2}$ Hubert J. Krenner, ${ }^{1,2,3}$ Sigmund Kohler, ${ }^{4}$ and Peter Hänggi ${ }^{1,2,3}$ \\ ${ }^{1}$ Institut für Physik, Universität Augsburg, Universitätsstraße 1, D-86153 Augsburg, Germany \\ ${ }^{2}$ Nanosystems Initiative Munich, Schellingstr. 4, D-80799 Munich, Germany \\ ${ }^{3}$ Center for NanoScience, Ludwig-Maximilians-Universität München, Geschwister-Scholl-Platz 1, D-80539 Munich, Germany \\ ${ }^{4}$ Instituto de Ciencia Materiales de Madrid, Consejo Superior de Investigaciones Cientficas, Cantoblanco, E-28049 Madrid, Spain
}

(Received 27 November 2013; published 24 January 2014)

\begin{abstract}
We explore the possibility of entangling an excitonic two-level system in a semiconductor quantum dot with a cavity defined on a photonic crystal by sweeping the cavity frequency across its resonance with the exciton transition. The dynamic cavity detuning is established by a radio frequency surface acoustic wave (SAW). It induces Landau-Zener transitions between the excitonic and the photonic degrees of freedom and thereby creates a superposition state. We optimize this scheme by using tailored Fourier-synthesized SAW pulses with up to five harmonics. The theoretical study is performed with a master equation approach for present state-of-the-art setups. Assuming experimentally demonstrated system parameters, we show that the composed pulses increase both the maximum entanglement and its persistence. The latter is only limited by the dominant dephasing mechanism, i.e., the photon loss from the cavity.
\end{abstract}

DOI: 10.1103/PhysRevA.89.012327

PACS number(s): 03.67.Bg, 42.50.Ct, 78.67.Hc, 71.36.+c

\section{INTRODUCTION}

Entanglement, an intrinsically quantum-mechanical correlation in composite systems, is indispensable for most quantum information protocols $[1,2]$ and, thus, should be available for any qubit realization. By definition, entanglement cannot be created by local operations and, thus, requires some interaction between the subsystems. In order to achieve a controlled degree of entanglement, one may turn on and off the effective interaction by tuning the subsystems into or close to resonance for a limited time. This includes a linear sweep across the resonance giving rise to a Landau-Zener (LZ) scenario at an avoided crossing. In between the regimes of adiabatic following and sudden switching, this process splits the wave function into two parts with a well-defined phase and thereby creates an entangled state.

Since entanglement relies on a well-defined phase relation, it is fundamentally limited by the susceptibility to decoherence of the chosen architecture. In the very active field of solidstate quantum systems the focus of LZ-based entanglement creation was set in the past mainly to superconducting [3,4] or spin-based [5] setups using their remarkable coherence properties. Although all-electrical radio frequency control can be readily implemented in these systems, transfer of the encoded quantum information to "flying" photonic qubits [6,7] at optical frequencies is extremely challenging. On the other hand, optically active quantum dot (QD) nanostructures provide a versatile platform allowing for tunable interdot coupling of exciton and spin states and for coupling excitonic two-level systems to optical resonators to implement a solidstate cavity QED system. For such optically active systems the implementation of LZ schemes has been considered extremely challenging, because most tuning mechanisms are quasistatic. While resonators available for cavity QED allow for reaching the required regime of strong light-matter interaction, the lifetime of cavity photons is typically more than two orders of magnitude shorter than the coherence times of excitons in an isotropic environment. Thus, theoretical proposals and experiments almost exclusively focused on quantum operations using the nonlinear optical properties of a static system.

In this paper we develop strategies to implement LZ gates in a semiconductor cavity QED system consisting of an excitonic two-level system in a single semiconductor QD coupled to a cavity localized in the optical mode of a photonic resonator (see Fig. 1). The time-dependent detuning is provided by surface acoustic waves (SAWs), for which we consider various feasible pulse shapes. In order to simulate the dynamics of the system, we numerically solve a master equation including the dissipative effects of (spontaneous) QD decay and photon loss. Moreover, we show that, for experimentally demonstrated system parameters, entanglement with a persistence of the photon lifetime can be achieved. Finally, we discuss directions to reduce the experimental complexity due to the high drive frequencies by using tailored Fourier-synthesized gating pulses.

\section{QUANTUM DOT IN A NANOCAVITY}

For the nanocavity we assume a high- $Q$ nanophotonic defect resonator defined in a two-dimensional photonic crystal membrane [8] interacting with a two-level system in a single semiconductor QD. This two-level system is formed by the crystal ground state of the QD and its fundamental optical excitation with one electron-hole pair, a single exciton $X$ [9]. Such systems have been studied over the past decade by several groups [10] who successfully demonstrated in key experiments both the weak-coupling [11-13] and the strong-coupling [14-19] regime of cavity QED. Most importantly, this type of nanocavity uniquely allows for a dynamic and reversible spectral control of the optical mode at gigahertz frequencies by the coherent acoustic phonon field of a radio frequency SAW [20], which is crucial for the implementation of LZ gates. We emphasize that the QD transition is sensitive to the strain and electric field of the SAW. Our previous experimental data [21] as well as other studies [22] on self-assembled InGaAs QDs suggest that the bandwidth of this modulation 
(a)

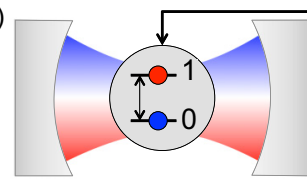

(b)
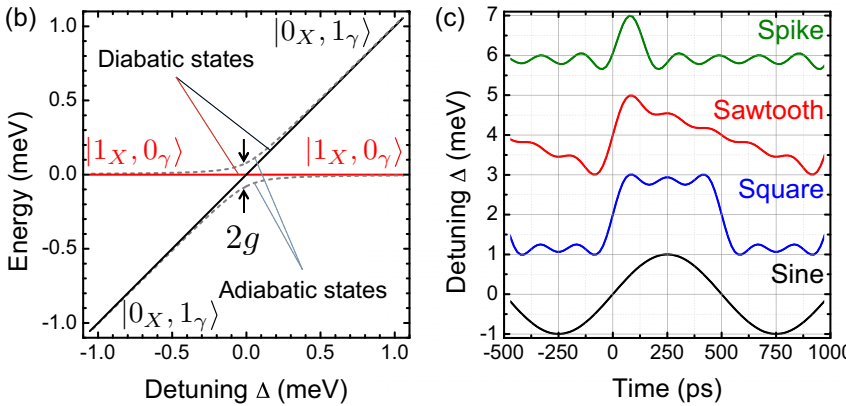

Time (ps)

FIG. 1. (Color online) (a) Semiconductor quantum dot placed in a photonic crystal, which serves as a realization for the cavity QED. Applying surface acoustic waves (SAWs) to the photonic crystal leads to a modulation of the cavity resonance frequency. (b) Energy spectrum of the system in dependence on the detuning $\Delta$. Owing to the cavity-QD coupling $g$, the exciton energy (black solid line) and the one-photon energy (red solid line) split at the degeneracy point $\Delta=0$ to form an avoided crossing (dotted lines). (c) Wave forms considered herein, which originate from a superposition of higher SAW harmonics.

is roughly three times smaller than that of the cavity, $\gamma$. Therefore, we set the QD transition to be constant during the acoustic cycle and treat the nanocavity resonance as time dependent. A schematic of this system is shown in Fig. 1(a).

For our modeling we restrict ourselves exclusively to $e x$ perimentally demonstrated system parameters for an InGaAsbased system with optical transitions at $E_{0}=\hbar \omega_{0}=1.3 \mathrm{eV}$ $\left(\lambda_{0}=954 \mathrm{~nm}, \omega_{0} / 2 \pi=314 \mathrm{THz}\right)$. First, for SAW excitation we assume $f_{\mathrm{SAW}} \leqslant 5 \mathrm{GHz}$. These frequencies are well below the highest frequencies reported for bulk GaAs- and GaAsbased suspended membranes of $f_{\mathrm{SAW}} \geqslant 24 \mathrm{GHz}$ [23] and $f_{\mathrm{SAW}}>9 \mathrm{GHz}$ [24], respectively. Second, for the coupled QD-cavity system, the limiting constituent is the photon loss rate $\Gamma_{\gamma}$ from the photonic crystal nanocavity. For our investigations we use $\hbar \Gamma_{\gamma}=25 \mu \mathrm{eV}\left(\Gamma_{\gamma}=38 \mathrm{GHz}\right)$, compatible with values reported by Ota et al. [19]. This value corresponds to a cavity quality factor of $Q=v / \Delta v=5.2 \times 10^{4}$ and a photon lifetime of $\tau_{\gamma}=Q / \omega_{0}=26 \mathrm{ps}$. The decay rate of the QD exciton $\hbar \Gamma_{X}=0.2 \mu \mathrm{eV}\left(\Gamma_{X}=300 \mathrm{MHz}\right)$ [17] is low when compared to $\Gamma_{\gamma}$. Furthermore, we assume the system in the strong-coupling regime with a vacuum Rabi splitting $\hbar g>$ $\hbar \Gamma_{\gamma}=25 \mu \mathrm{eV}$. The energies of the adiabatic and nonadiabatic states of this coupled system are plotted in Fig. 1(b) as a function of detuning $\Delta$. The adiabatic energies show the characteristic avoided crossing between $X$ and $\gamma$. We can write the corresponding states $\left|n_{X}, n_{\gamma}\right\rangle$, where $n_{X}=0$ and 1 correspond to the two states of the two-level system, while $n_{\gamma}$ refers to the cavity photon number. For adiabatically slow sweeping, the exciton state $\left|1_{X}, 0_{\gamma}\right\rangle$ transforms into a photon, $\left|0_{X}, 1_{\gamma}\right\rangle$, by adiabatic following. Increasing the sweep velocity, the initial states remain partially occupied with a probability given by the LZ formula [25-28]:

$$
P_{\mathrm{LZ}}=1-\exp \left(-2 \pi g^{2} / \hbar v\right)
$$

where the coupling $g$ determines the splitting at the degeneracy point $\Delta=0$. In the derivation of Eq. (1), it was assumed that the detuning $\Delta(t)=v t$ varies at a constant rate $v$ which for our SAW-based approach is proportional to the SAW frequency and SAW amplitude, $v \propto f_{\mathrm{SAW}} A$. Since we restrict ourselves to experimentally accessible parameters, we keep the amplitude of the SAW modulation constant throughout the paper to $A=\max (\Delta)=1 \mathrm{meV}$, a base frequency of $f_{0}=$ $1 \mathrm{GHz}$, and wave forms Fourier synthesized by superpositions of harmonics: In addition to pure sine drive at $f_{\mathrm{SAW}}=n f_{0}$ and $n=1,3$, and 5 , we investigate the three synthesized wave forms summarized in Fig. 1(c). In the limit $n \rightarrow \infty$, they converge either to a $\delta$ peak, henceforth referred to as spike, or to a wave form of either square or sawtooth type, respectively. For a realistic modeling, these waves consist of the fundamental frequency $f_{0}$ and its first four harmonics. For the LZ gate, the system is initialized by a Rabi oscillation from its ground state to the excited state $X$ by a laser pulse $[9,29]$. To ensure selective excitation of $X$ and to avoid initial photon population in the cavity the spectral bandwidth of this excitation pulse has to be $\delta E_{\text {laser }} \leqslant 250 \mu \mathrm{eV}$ corresponding to a pulse length $\delta t_{\text {laser }} \geqslant 20.7 \mathrm{ps}$. This in turn requires that the initialization has to occur at $t_{0}>\delta t_{\text {laser }}$ before the system is tuned through resonance, at which the initial product state $\left|1_{X}, 0_{\gamma}\right\rangle$ is converted to an entangled state.

\section{A. Excitonic quantum dot coupled to a SAW-driven cavity}

Without the SAW driving, the system sketched in Fig. 1(a) is described by the Jaynes-Cummings (JC) Hamiltonian [30]:

$$
H_{\mathrm{JC}}=\frac{\varepsilon}{2} \sigma_{z}+\hbar \omega_{0} a^{\dagger} a+g\left(a \sigma_{+}+a^{\dagger} \sigma_{-}\right),
$$

where the pseudospin operators $\sigma_{z}$ and $\sigma_{ \pm}$describe the excitonic quantum dot within a two-level approximation in the basis of the ground state $\left|0_{X}\right\rangle$ and the one-exciton state $\left|1_{X}\right\rangle$ with the energy splitting $\varepsilon$. The bosonic operators $a$ and $a^{\dagger}$ refer to the cavity with resonance frequency $\omega_{0}$, which is dipole coupled to the quantum dot according to $g \sigma_{x}\left(a^{\dagger}+a\right)$. Close to resonance, i.e., for $\varepsilon \approx \omega_{0}$, we can neglect the counter-rotating terms $a \sigma_{-}$and $a^{\dagger} \sigma_{+}$to obtain the last term of the Hamiltonian Eq. (2). Then the Hilbert space of the composed system discerns into doublets spanned by the states $\left|1_{X}, n_{\gamma}\right\rangle$ and $\left|0_{X},(n+1)_{\gamma}\right\rangle$. As a function of the detuning $\Delta=\varepsilon-\hbar \omega_{0}$, the eigenenergies of $H_{\mathrm{JC}}$ form avoided crossings of width $2 \sqrt{n+1} g$ [31] [see Fig. 1(b)]. The central idea is to exploit the LZ dynamics at the avoided crossing of the lowest doublet to entangle the quantum dot with the cavity, i.e., to reach a final state $\propto\left|1_{X}, 0_{\gamma}\right\rangle+e^{i \varphi}\left|0_{X}, 1_{\gamma}\right\rangle$ with a well-defined but possibly unknown and time-dependent phase $\varphi$.

The SAW modulates the cavity frequency so that it becomes time dependent, i.e., $\omega_{0} \rightarrow \omega_{0}(t)$. This implies that also the detuning gets modulated with time: $\Delta \rightarrow \Delta(t)$. For the case of a sinusoidal wave it is $\Delta(t)=\Delta_{0}+A \sin \left[\Omega\left(t-t_{0}\right)\right]$, where the amplitude $A$ has to exceed the static detuning $\Delta_{0}$ to pass through the avoided crossing. If the amplitude is large compared to the crossing region, the driving can be linearized, resulting in a sweep velocity of the order $v \sim A \Omega$. This sweep velocity is of crucial importance because, according to the LZ formula Eq. (1), it determines the probability 
for adiabatically following the ground state. Significant exciton-cavity entanglement requires $P_{\mathrm{LZ}} \approx 1 / 2$. On the other hand, it is desirable to slow down the modulation after the entanglement is created, so that further state manipulations or a readout of the quantum state can be performed. Therefore we like to exploit recent experimental achievements of a controlled superposition of higher harmonics to the SAW and consider more generic waves that lead to the detuning $\Delta(t)=$ $\Delta_{0}+\sum_{n=1}^{N} A_{n} \sin \left[n \Omega\left(t-t_{0}\right)+\phi_{n}\right]$. In an experiment both the amplitudes $A_{n}$ and the phases $\phi_{n}$ can be controlled rather well, which enables a flexible design of the pulses. Here we consider, besides purely sinusoidal driving, also waves with the characteristic shapes of a square, a sawtooth, and a spike. We restrict ourselves to the experimentally feasible case in which those waves are approximated by a fundamental angular frequency of $\Omega=2 \pi \times 1 \mathrm{GHz}$ and its harmonics up to order $N=5$; i.e., we consider the drivings

$$
\Delta(t)=\Delta_{0}+ \begin{cases}A \sin \left[\Omega\left(t-t_{0}\right)\right] & \text { sine, } \\ \sum_{n=0}^{5} \frac{A}{N} \cos \left[n \Omega\left(t-t_{0}\right)\right] & \text { spike, } \\ \sum_{n=0}^{2} \frac{A}{2 n+1} \sin \left[n \Omega\left(t-t_{0}\right)\right] & \text { square, } \\ \sum_{n=1}^{5} \frac{A}{n} \sin \left[n \Omega\left(t-t_{0}\right)\right] & \text { sawtooth, }\end{cases}
$$

sketched in Fig. 1(c). Moreover, in order to highlight the benefit of nonsinusoidal pulses, we also consider pure sine waves with the angular frequencies $3 \Omega$ and $5 \Omega$.

\section{B. Decoherence and master equation}

Entanglement is a genuine quantum feature and, thus, is rather sensitive to decoherence caused by the interaction with environmental degrees of freedom. In our case the latter are mainly the photonic modes $v$ outside the cavity. Their influence can be modeled by the system-bath Hamiltonian $H_{\mathrm{env}}=\sum_{v} \hbar \omega_{\nu} a_{v}^{\dagger} a_{v}+Z \sum_{\nu} \lambda_{v}\left(a_{v}^{\dagger}+a_{v}\right)$, where $\omega_{v}$ denotes the frequency of mode $v$, while $\lambda_{v}$ is the coupling strength to a system operator $Z$ which here is the cavity dipole operator $Z_{\gamma}=a^{\dagger}+a$. For a later continuum limit, we assume for the coupling the Ohmic spectral density $J(\omega)=\pi \sum_{\nu}\left|\lambda_{\nu}\right|^{2} \delta(\omega-$ $\left.\omega_{\nu}\right) \equiv \pi \alpha_{\gamma} \omega / 2$ with the dimensionless dissipation parameter $\alpha_{\gamma}$ [32-34]. Under the condition that the environment is initially in a Gibbs state, we derive for the reduced system density operator $\rho$ a Bloch-Redfield master equation $[35,36]$. Moreover, we take also dissipative transitions of the quantum dot into account, which we model in the same way but with the excitonic dipole moment $Z_{X}=\sigma_{+}+\sigma_{-}$. For consistency with the system Hamiltonian Eq. (2), we apply a rotating-wave approximation to the master equation such that we finally arrive at $[30,31]$

$$
\frac{d}{d t} \rho=-\frac{i}{\hbar}\left[H_{\mathrm{JC}}(t), \rho\right]+\mathcal{L}_{\gamma}(\rho)+\mathcal{L}_{X}(\rho),
$$

where the Lindblad forms

$$
\begin{gathered}
\mathcal{L}_{\gamma}(\rho)=\frac{\Gamma_{\gamma}}{2}\left(2 a \rho a^{\dagger}-a^{\dagger} a \rho-\rho a^{\dagger} a\right), \\
\mathcal{L}_{X}(\rho)=\frac{\Gamma_{X}}{2}\left(2 \sigma_{-} \rho \sigma_{+}-\sigma_{+} \sigma_{-} \rho-\rho \sigma_{+} \sigma_{-}\right)
\end{gathered}
$$

describe cavity and quantum dot dissipation, respectively. The decay rates $\Gamma_{\gamma}$ and $\Gamma_{X}$ are determined by $J(\omega)$ evaluated at the transitions frequencies of the dot-cavity Hamiltonian Eq. (2). Owing to the smallness of the detuning $|\Delta| \ll \varepsilon, \omega_{0}(t)$ and the smooth Ohmic spectral density, we can ignore the energy shifts induced by the dot-cavity coupling $g$, so that we can evaluate the spectral densities at the bare cavity and exciton frequency to obtain $\Gamma_{\gamma}=\pi \alpha_{\gamma} \omega_{0}$ and $\Gamma_{X}=\pi \alpha_{X} \varepsilon$, respectively. Notice that we have assumed that the environmental temperature is rather low, such that thermal excitation is negligible. Then the final state of the time-independent problem is the ground state $\left|0_{X}, 0_{\gamma}\right\rangle$, also beyond the rotating-wave approximation [37].

\section{ENTANGLEMENT DYNAMICS}

We consider the dynamics of the cavity-QD setup after an exciton is created at time $t=0$, while the cavity is empty; i.e., we numerically integrate the master equation starting with the initial state $\left|1_{X}, 0_{\gamma}\right\rangle$. In the course of time, the SAW sweeps the energies of the two subsystems over an avoided crossing with the state $\left|0_{X}, 1_{\gamma}\right\rangle$ reached at $t \approx t_{0}$. Moreover, the Lindblad terms cause a decay toward the ground state $\left|0_{X}, 0_{\gamma}\right\rangle$. Our main aim is to investigate and to optimize the degree of entanglement for differently shaped SAW pulses. In order to quantify the entanglement, we treat the cavity within two-level approximation in the subspace spanned by the states $\left|0_{\gamma}\right\rangle$ and $\left|1_{\gamma}\right\rangle$ with the corresponding Pauli matrices $\sigma_{y}^{\gamma}$. This approximation is well justified, because our Hamiltonian Eq. (2) preserves the total number of excitations, while our
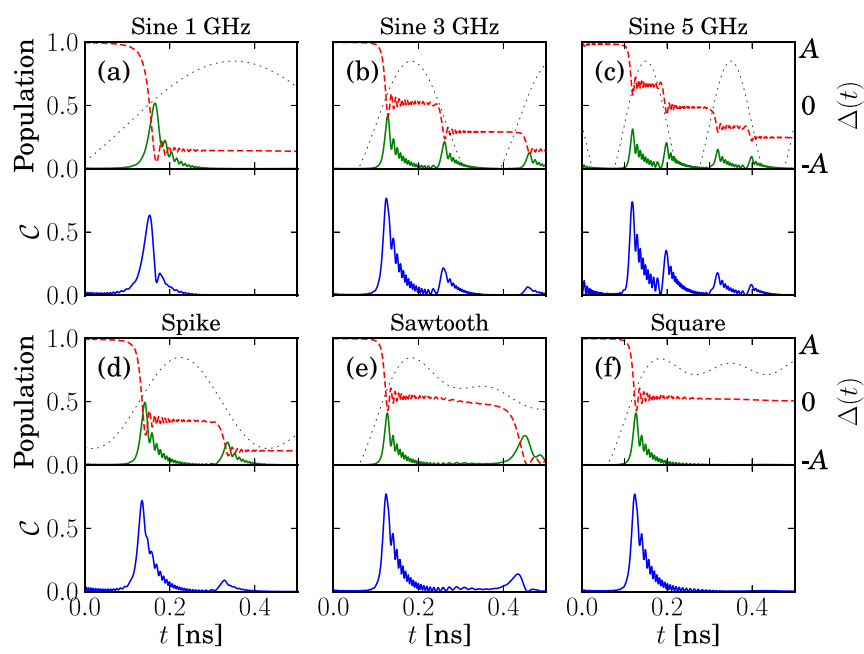

FIG. 2. (Color online) LZ entanglement dynamics for SAW shapes of a pure sine with frequencies $1 \mathrm{GHz}$ (a), $3 \mathrm{GHz}$ (b), and $5 \mathrm{GHz}$ (c), as well as for a spike (d), a sawtooth (e), and a square (f), each with fundamental frequency $\Omega / 2 \pi=1 \mathrm{GHz}$. The cavity-dot coupling is $g=35 \mu \mathrm{eV}$, while the static detuning $\Delta_{0}=0.3 \mathrm{meV}$ is modulated with an amplitude $A=1 \mathrm{meV}$, such that the crossing is reached at time $t_{0}=T / 10=0.5 \mathrm{~ns}$. The cavity and exciton decay rates read $\Gamma_{\gamma}=25 \mu \mathrm{eV} / \hbar$ and $\Gamma_{X}=0.2 \mu \mathrm{eV} / \hbar$. Upper panels: Population of the states $\left|1_{X}, 0_{\gamma}\right\rangle$ (red solid line) and $\left|0_{X}, 1_{\gamma}\right\rangle$ (green dashed line). The dotted line visualizes the course of the detuning $\Delta(t)$. Lower panels: Cavity-dot entanglement in terms of the concurrence $\mathcal{C}$. 
low-temperature dissipation kernels $\mathcal{L}_{\gamma}$ and $\mathcal{L}_{X}$ only contain decay terms. Then we can employ as entanglement measure the concurrence [38,39]

$$
\mathcal{C}(\rho)=\max \left(\chi_{1}-\chi_{2}-\chi_{3}-\chi_{4}, 0\right),
$$

where the $\chi_{i}$ are the square roots of the eigenvalues of the matrix $\rho \sigma_{y} \sigma_{y}^{\gamma} \rho^{*} \sigma_{y} \sigma_{y}^{\gamma}$ in descending order. Here $\rho^{*}$ denotes the complex conjugate of the density matrix expressed in a basis of Bell states [38].

In order to get a first impression of the dynamics, we depict in Fig. 2 the time evolution of the populations of the states $\left|1_{X}, 0_{\gamma}\right\rangle$ and $\left|0_{X}, 1_{\gamma}\right\rangle$ and the corresponding entanglement for various wave forms, while all other parameters are set equal. For a purely sinusoidal driving with frequency $\Omega / 2 \pi=1 \mathrm{GHz}$, the population of the initial state is by and large transferred to $\left|0_{X}, 1_{\gamma}\right\rangle$. This corresponds to imperfect adiabatic following. At an intermediate stage at time $t \approx t_{0}$, the populations of both states are comparable, while phase coherence between the participating states ensures good entanglement with a concurrence up to $\mathcal{C} \approx 0.7$. However, since for these parameters $P_{\mathrm{LZ}}$ is significantly larger than $1 / 2$, soon after the crossing the one-photon state becomes highly populated. Therefore the systems disentangle soon after having passed the crossing. Thus, we must increase the sweep velocity, which can be achieved by using a higher frequency. The results in panels (b) and (c) demonstrate that this can indeed augment the concurrence. Moreover, it increases the time during which the concurrence exceeds a certain threshold value. This "entanglement persistence" is mainly limited by the cavity decay rate $\Gamma_{\gamma}$, at least under the realistic condition $\Gamma_{\gamma} \gg \Gamma_{X}$. Thus, our goal is to find parameters and wave forms for which a significant entanglement is present during a time of the order $1 / \Gamma_{\gamma}$. A theoretically interesting observation is that for higher frequencies the system may pass through the avoided crossing several times in the time range considered. The resulting repeated passages depend on the phase acquired in between the crossings, leading to Landau-Zener-Stückelberg interference [26-28,40]. However, for realistic cavity decay rates, dephasing is too fast and, thus, the coherent superposition of our entangled states turns into a (separable) mixture. Therefore, we will not further discuss interference effects. Even though SAWs with frequencies of 3 or $5 \mathrm{GHz}$ are feasible, inducing them with a large intensity such that the detuning amplitude becomes $1 \mathrm{meV}$ represents a rather difficult task. Waves with lower amplitudes are not helpful, because they lead to smaller sweep velocities and, thus, we would lose what we gained from the higher frequency. Moreover, the initial preparation of the exciton must be performed during a fraction of the driving period, because it takes a certain time and cannot be triggered with arbitrary precision. Thus, for shorter driving periods, one will encounter difficulties to carry out the preparation. These difficulties can be circumvented by employing more elaborate pulses such as the ones sketched in Fig. 1(c) and mathematically expressed in Eq. (3). Notice that for these pulses the contribution of each harmonic is significantly smaller than $1 \mathrm{meV}$, while the driving period remains at $2 \pi / \Omega=1 \mathrm{~ns}$. The resulting entanglement dynamics is plotted in Figs. 2(e) and 2(f). As compared to panel (a), the performance of the entanglement creation has improved. Moreover, as we will see below, this performance can be reached in a broader parameter range.

In an experimental implementation of our proposed scheme, one would on the one hand like to obtain a rather large maximum for the concurrence, while on the other hand an appreciable entanglement should be found during a not too short time, ideally limited only by the cavity decay $\Gamma_{\gamma}$. Moreover, the cavity-dot coupling $g$ is essentially a fixed parameter determined during chip fabrication, which implies that the width of the avoided crossing can be tuned only within a narrow range via the driving frequency and the amplitude.
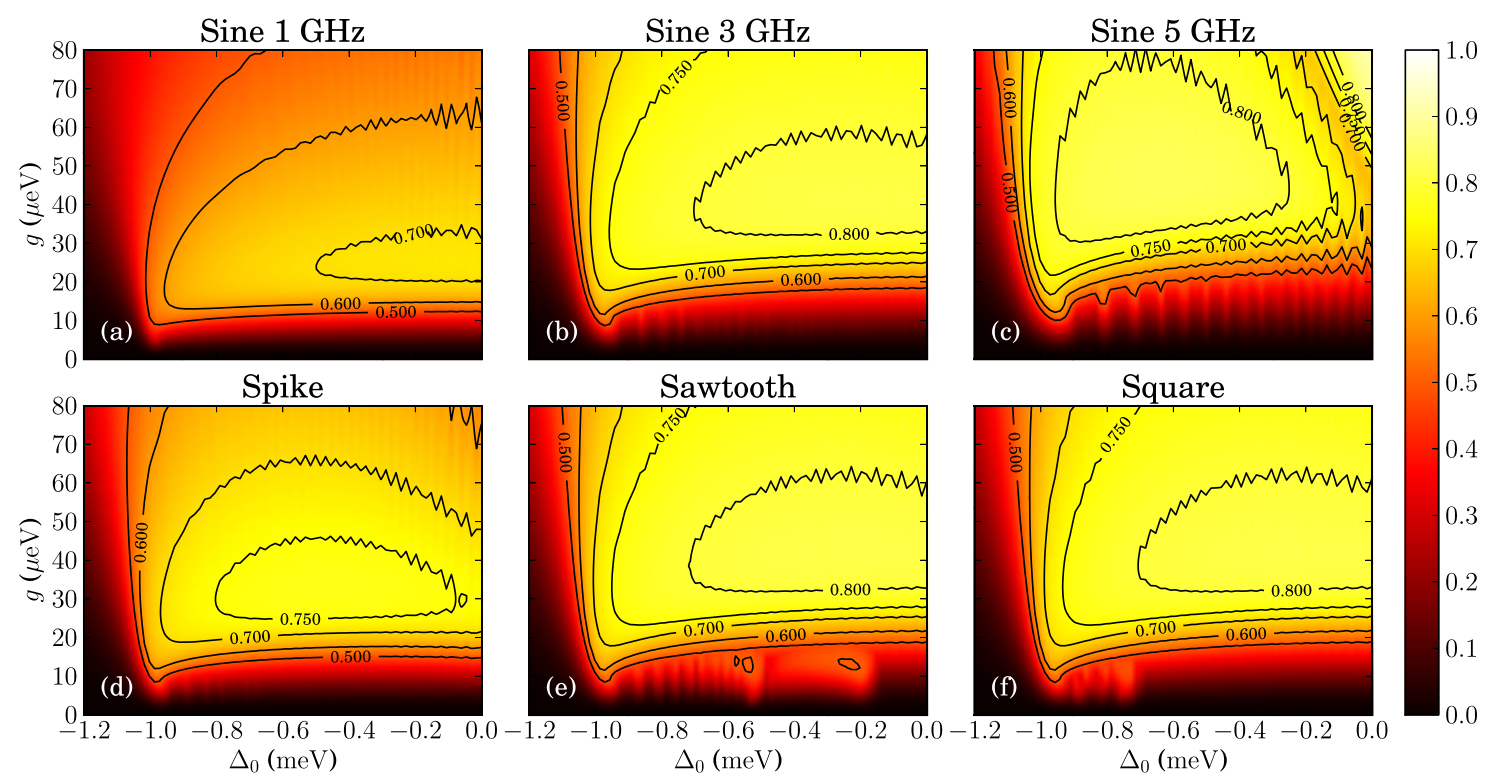

FIG. 3. (Color online) Maximum of the concurrence achieved as a function of the static detuning $\Delta_{0}$ and the dot-cavity coupling $g$. All other parameters, the wave forms, and the arrangement of the panels are as in Fig. 2. 

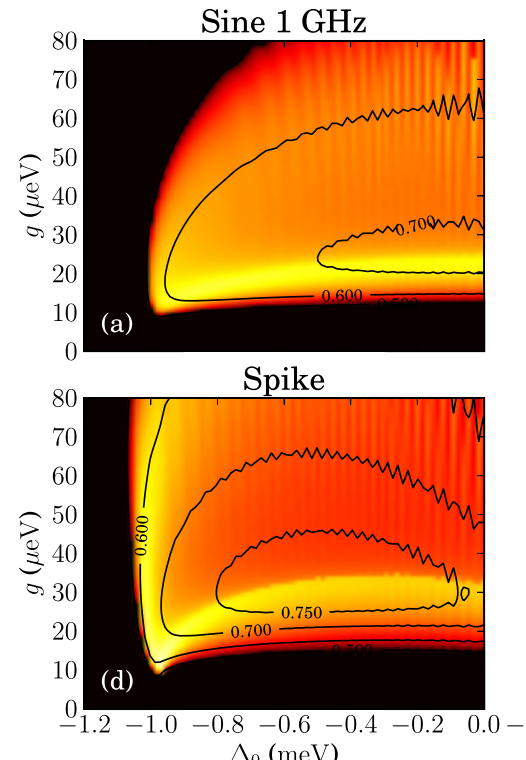

$\Delta_{0}(\mathrm{meV})$
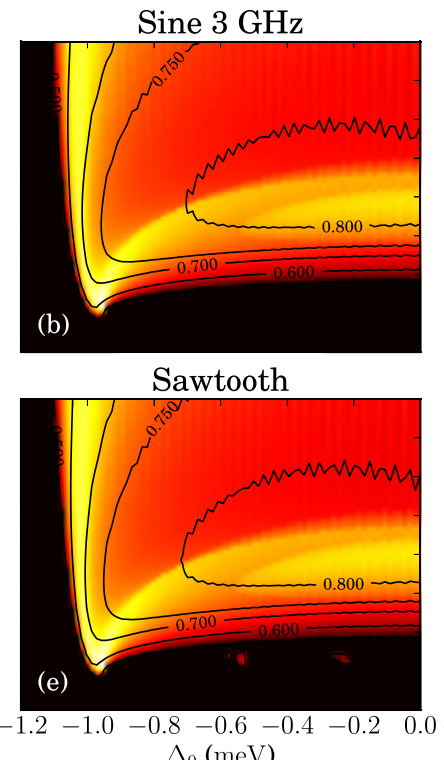

$\Delta_{0}(\mathrm{meV})$

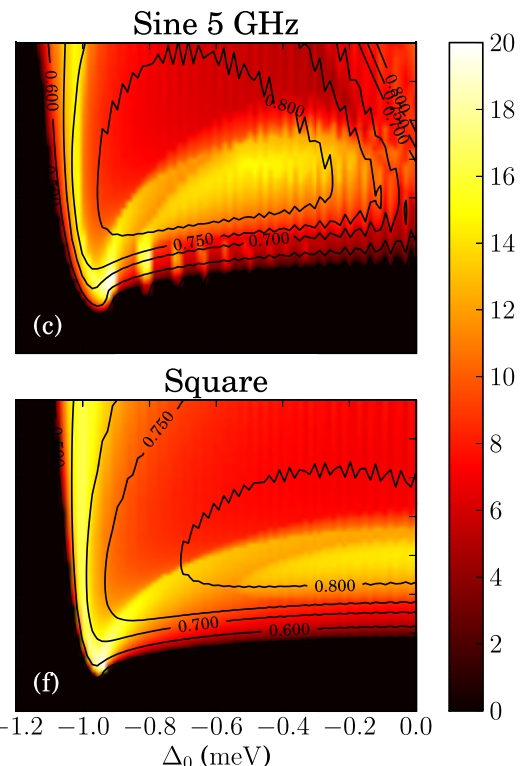

$\Delta_{0}(\mathrm{meV})$

FIG. 4. (Color online) Persistence of the entanglement, i.e., time during which the concurrence exceeds the threshold value set by $\mathcal{C}>1 / 2$. All parameters, wave forms, and the contour lines marking the maximum of the concurrence are as in Fig. 3 . The unit of the color bar is in ps.

Therefore, it is desirable that the results do not depend too sensitively on $g$. Given this low flexibility, suggestions for more promising SAW shapes are particularly welcome.

In order to characterize the performance of each wave form, we employ two figures of merit. The first one is the maximal concurrence $\mathcal{C}$ reached during a time $T / 4$ centered at the avoided crossing as function of $g$ and $\Delta_{0}$, depicted in Fig. 3. For all frequencies and pulse shapes considered, the concurrence can reach values up to $\mathcal{C} \approx 0.8$. However, the basic sinusoidal wave at $1 \mathrm{GHz}$ yields this value only in a small range of the coupling $g$, which requires a precise fabrication process. Since, as discussed above, for 3 and $5 \mathrm{GHz}$, the required amplitudes of the order $1 \mathrm{meV}$ are difficult to achieve, the more elaborate pulse shapes are clearly preferable. For all three composed pulses, the plots of the concurrence maximum behave very similarly. The common feature of all three waves is the rather steep slope of the detuning $\Delta(t)$ close to the center of the avoided crossing, as can be appreciated in the upper panels of Fig. 2. This suggests that the main effect of the higher-order Fourier components is to augment the sweep velocity at the crossing.

Our second figure of merit is the persistence time $\tau$ of the entanglement defined as the time during which $\mathcal{C}>1 / 2$. This quantifier is depicted in Fig. 4. The contour lines enable a comparison with the results shown in Fig. 3. This reveals that a large maximum does not necessarily coincide with long persistence. This is particularly the case for the sinusoidal pulses with higher frequencies [panels (b) and (c)]. Nevertheless there exist regimes where both the entanglement maximum and the persistence are rather favorable and where $\tau$ practically reaches its theoretical limit, which is the cavity lifetime $1 / \Gamma_{\gamma}=26 \mathrm{ps}$. As for the maximum, the plot for the sine wave with $3 \mathrm{GHz}$ and the ones for the composed pulses [panels (d)-(f)] look similar. However, the former has the disadvantage of being experimentally more demanding. Interestingly enough, in two regimes, albeit small, pure sine waves yield surprisingly long entanglement duration: First, for $1 \mathrm{GHz}$ in the regime $\Delta_{0} \approx-1 \mathrm{meV}, g \gtrsim 10 \mu \mathrm{eV}$, where, however, the maximum is rather low; second, for $5 \mathrm{GHz}$, we witness in panel (f) at $g \approx 25 \mu \mathrm{eV}$ some islands with $\tau \gtrsim 20$ ps. Combining the two criteria of large maximum $\mathcal{C}$ and long persistence, we can conclude that for an experimental realization the quantum dot-cavity coupling $g$ should be in the range $20-50 \mu \mathrm{eV}$.

\section{DISCUSSION AND CONCLUSION}

We have preformed a theoretical study demonstrating the experimental feasibility of entanglement generation in a semiconductor QD-nanocavity system by a SAW-gated LZ transition. Using exclusively experimentally demonstrated state-of-the-art system parameters we find high levels of entanglement corresponding to a concurrence of $\mathcal{C}>0.8$. Its persistence is mainly limited by the photon loss from the cavity. This dominant dephasing mechanism arises from the moderate quality factor of such semiconductor-based cavities. An extension of this scheme to Fourier-synthesized SAW wave forms promises two significant advantages over a single frequency sinusoidal drive. First, our model predicts for square and sawtooth pulses a large concurrence over the system-limited time scales for a broad range of $g$ and $\Delta_{0}$. The second advantage lies in the experimental implementation: The complexity to achieve a sufficiently large modulation amplitude of $1 \mathrm{meV}$ increases significantly with increasing $f_{\mathrm{SAW}}$, in particular for modulation frequencies of $3 \mathrm{GHz}$ and higher. In contrast, Fourier-synthesized wave forms with only moderate amplitudes of higher harmonics significantly overcompensate the additional requirements for the design of the transducers for SAW generation. Moreover, the fundamental SAW period in the experiment remains constant at $1 \mathrm{~ns}$, which facilitates the synchronization with the optical initialization and measurement of the entanglement. The latter can be implemented, e.g., by extending existing schemes 
based on reflectivity spectroscopy [16] using short ( $<1 \mathrm{ps)}$ and broadband laser pulses as a function of time during the acoustic cycle. Moreover, since the system is initialized in the exciton state, i.e., in the lower branch of the avoided crossing, any signal from the upper branch detected in the loss spectrum provides a fingerprint of the LZ transition. Finally we want to note that the results of our theoretical study can be directly transferred to other types of semiconductor cavities, most notably Bragg-type microcavities [41], which are SAW compatible [42]. In addition, among the broad variety of control techniques, electrical tuning of the QD transition via the quantum confined Stark effect [17,43-45] could be an alternative approach to realize the required gigahertz frequencies.

\section{ACKNOWLEDGMENTS}

We gratefully acknowledge financial support by the Deutsche Forschungsgemeinschaft (DFG) via Sonderforschungsbereich SFB 631 (Projects No. A5 and No. B5) and the Emmy Noether Program (HJK, KR3790/2-1). This work was supported by the Spanish Ministry of Economy and Competitiveness via Grant No. MAT2011-24331.
[1] M. A. Nielsen and I. L. Chuang, Quantum Computing and Quantum Information (Cambridge University Press, Cambridge, 2000).

[2] R. Horodecki, P. Horodecki, M. Horodecki, and K. Horodecki, Rev. Mod. Phys. 81, 865 (2009).

[3] K. Saito, M. Wubs, S. Kohler, P. Hänggi, and Y. Kayanuma, Europhys. Lett. 76, 22 (2006).

[4] M. Wubs, S. Kohler, and P. Hänggi, Physica E 40, 187 (2007).

[5] H. Ribeiro and G. Burkard, Phys. Rev. Lett. 102, 216802 (2009).

[6] J. I. Cirac, P. Zoller, H. J. Kimble, and H. Mabuchi, Phys. Rev. Lett. 78, 3221 (1997).

[7] W. Yao, R.-B. Liu, and L. J. Sham, Phys. Rev. Lett. 95, 030504 (2005).

[8] Y. Akahane, T. Asano, B. S. Song, and S. Noda, Nature (London) 425, 944 (2003).

[9] A. Zrenner, E. Beham, S. Stufler, F. Findeis, M. Bichler, and G. Abstreiter, Nature (London) 418, 612 (2002).

[10] S. Noda, M. Fujita, and T. Asano, Nat. Photon. 1, 449 (2007).

[11] D. Englund, D. Fattal, E. Waks, G. Solomon, B. Zhang, T. Nakaoka, Y. Arakawa, Y. Yamamoto, and J. Vučković, Phys. Rev. Lett. 95, 013904 (2005).

[12] A. Kress, F. Hofbauer, N. Reinelt, M. Kaniber, H. J. Krenner, R. Meyer, G. Böhm, and J. J. Finley, Phys. Rev. B 71, 241304 (2005).

[13] W.-H. Chang, W.-Y. Chen, H.-S. Chang, T.-P. Hsieh, J.-I. Chyi, and T.-M. Hsu, Phys. Rev. Lett. 96, 117401 (2006).

[14] T. Yoshie, A. Scherer, J. Hendrickson, G. Khitrova, H. M. Gibbs, G. Rupper, C. Ell, O. B. Shchekin, and D. G. Deppe, Nature (London) 432, 200 (2004).

[15] K. Hennessy, A. Badolato, M. Winger, D. Gerace, M. Atatüre, S. Gulde, S. Fält, E. L. Hu, and A. Imamoğlu, Nature (London) 445, 896 (2007).

[16] D. Englund, A. Faraon, I. Fushman, N. Stoltz, P. Petroff, and J. Vučković, Nature (London) 450, 857 (2007).

[17] A. Laucht, N. Hauke, J. M. Villas-Bôas, F. Hofbauer, G. Böhm, M. Kaniber, and J. J. Finley, Phys. Rev. Lett. 103, 087405 (2009).

[18] S. M. Thon, M. T. Rakher, H. Kim, J. Gudat, W. T. M. Irvine, P. M. Petroff, and D. Bouwmeester, Appl. Phys. Lett. 94, 111115 (2009).

[19] Y. Ota, S. Iwamoto, N. Kumagai, and Y. Arakawa, Phys. Rev. Lett. 107, 233602 (2011).

[20] D. A. Fuhrmann, S. M. Thon, H. Kim, D. Bouwmeester, P. M. Petroff, A. Wixforth, and H. J. Krenner, Nat. Photon. 5, 605 (2011).

[21] F. J. R. Schülein, K. Müller, M. Bichler, G. Koblmüller, J. J. Finley, A. Wixforth, and H. J. Krenner, Phys. Rev. B 88, 085307 (2013).
[22] J. R. Gell, M. B. Ward, R. J. Young, R. M. Stevenson, P. Atkinson, D. Anderson, G. A. C. Jones, D. A. Ritchie, and A. J. Shields, Appl. Phys. Lett. 93, 81115 (2008).

[23] I. V. Kukushkin, J. H. Smet, L. Hoppel, U. Waizmann, M. Riek, W. Wegscheider, and K. von Klitzing, Appl. Phys. Lett. 85, 4526 (2004).

[24] Y. Takagaki, E. Wiebicke, P. V. Santos, R. Hey, and K. H. Ploog, Semicond. Sci. Technol. 17, 1008 (2002).

[25] L. D. Landau, Physik. Zeitschr. Sowjetunion 2, 46 (1932).

[26] C. Zener, Proc. R. Soc. London A 137, 696 (1932).

[27] E. C. G. Stückelberg, Helv. Phys. Acta 5, 369 (1932).

[28] E. Majorana, Nuovo Cimento 9, 43 (1932).

[29] H. J. Krenner, S. Stufler, M. Sabathil, E. C. Clark, P. Ester, M. Bichler, G. Abstreiter, J. J. Finley, and A. Zrenner, New J. Phys. 7, 184 (2005).

[30] C. W. Gardiner, Quantum Noise, Springer Series in Synergetics Vol. 56 (Springer, Berlin, 1991).

[31] D. F. Walls and G. J. Milburn, Quantum Optics, 2nd ed. (Springer, Heidelberg, 1995).

[32] A. J. Leggett, S. Chakravarty, A. T. Dorsey, M. P. A. Fisher, A. Garg, and W. Zwerger, Rev. Mod. Phys. 59, 1 (1987).

[33] P. Hänggi, P. Talkner, and M. Borkovec, Rev. Mod. Phys. 62, 251 (1990).

[34] U. Weiss, Quantum Dissipative Systems, 2nd ed. (World Scientific, Singapore, 1998).

[35] A. G. Redfield, IBM J. Res. Develop. 1, 19 (1957).

[36] K. Blum, Density Matrix Theory and Applications, 2nd ed. (Springer, New York, 1996).

[37] J. Thingna, J. S. Wang, and P. Hänggi, J. Chem. Phys. 136, 194110 (2012).

[38] S. Hill and W. K. Wootters, Phys. Rev. Lett. 78, 5022 (1997).

[39] W. K. Wootters, Phys. Rev. Lett. 80, 2245 (1998).

[40] S. N. Shevchenko, S. Ashhab, and F. Nori, Phys. Rep. 492, 1 (2010).

[41] J. P. Reithmaier, G. Sek, A. Löffler, C. Hofmann, S. Kuhn, S. Reitzenstein, L. V. Keldysh, V. D. Kulakovskii, T. L. Reinecke, and A. Forchel, Nature (London) 432, 197 (2004).

[42] M. M. de Lima, Jr. and P. V. Santos, Rep. Prog. Phys. 68, 1639 (2005).

[43] A. Faraon, A. Majumdar, H. Kim, P. Petroff, and J. Vučković, Phys. Rev. Lett. 104, 047402 (2010).

[44] C. Kistner, S. Reitzenstein, C. Schneider, S. Höfling, and A. Forchel, Appl. Phys. Lett. 94, 221103 (2009).

[45] M. T. Rakher, N. G. Stoltz, L. A. Coldren, P. M. Petroff, and D. Bouwmeester, Phys. Rev. Lett. 102, 097403 (2009). 